\title{
An Improved Lyapunov Function Based Stability Analysis Method for Fuzzy Logic Control Systems
}

\author{
L. K. Wong \\ Frank H. F. Leung \\ Peter K. S. Tam \\ Department of Electronic and Information Engineering, \\ The Hong Kong Polytechnic University, \\ Hung Hom, Hong Kong.
}

\begin{abstract}
Many existing stability analysis methods for fuzzy logic control systems like TS fuzzy model based methods usually tackle plants that are linear with respect to control. This paper proposes an improved method which does not have such restriction. The proposed method employs a Lyapunov function to prove the stability of the non-linear fuzzy logic control system. An illustrative example will be given to demonstrate the ability of the method. The plant in this example has a control signal function composed of piecewise linear functions, saturation functions and a deadband.
\end{abstract}

\section{INTRODUCTION}

A fuzzy logic control system consists of a plant and a fuzzy logic controller (FLC). As shown in Fig. 1, the output of the FLC is a function of the degrees of membership of the fuzzy rules, and these degrees of membership are function of input variable(s), e.g. system states. The control system becomes highly non-linear. The analysis of system stability for this system is a difficult task.

A common approach to tackle the stability problem of fuzzy logic control systems is based on TS fuzzy plant models. The plant of the control system is first represented by a TS fuzzy model. Then an FLC, in which linear state feedback is usually used in the output of every fuzzy rule, is designed. Tanaka et al $[7,8]$ applied the parallel distributed compensation technique to develop the FLC. Then LMI techniques were employed to find a common Lyapunov function so that the system stability is ensured. Cao et al [46] decomposed the fuzzy plant model into many sub-systems, each of them consists of a nominal system and interactions of the sub-systems. Then a design method was proposed that can give an FLC capable of stabilizing the system. Other stability analysis methods involve TS fuzzy plant models can be found in the literature [12-13].

There were also studies on analysing fuzzy logic control systems which do not use fuzzy plant models [9-11]. Some methods use error and change of error as inputs of the FLC. Then the state plane is partitioned and analysis is carried out in every partition individually. However, these methods may become difficult to apply when high order systems are involved. Also, the input membership functions are usually required to have a regular shape with an even distribution in the domain. In this case, the flexibility of designing the FLC is reduced.

Recently, we have proposed a stability analysis method which employs Lyapunov direct method for fuzzy logic control systems [1-2]. The proposed method handles every fuzzy sub-system (that will be defined in section 2.1) individually instead of the system as a whole in order to avoid dealing with the complex non-linear control signal generated by the FLC. This method does not require a fuzzy plant model, and the shape and distribution of the input membership functions are not restricted to certain patterns.

In this paper, we further develop this stability analysis method so that it is not restricted to systems that are linear with respect to control [14]. If the plant is of the form $\dot{\boldsymbol{x}}=\boldsymbol{f} \boldsymbol{x})+\boldsymbol{b}(\boldsymbol{x}) g(u)+\boldsymbol{w}$, where $g(u)$ is a scalar linear or nonlinear function of $u$, and is either a monotonic increasing or a monotonic decreasing function, the proposed stability analysis method can be applied. It should be noted that the methods based on TS fuzzy plant model usually deal with plants with $u$ instead of $g(u)$. It is because a plant with $g(u)$ may lead to a premise depending on $u$ (in addition to the system state) in the fuzzy control rules. Then the design of the FLC and the stability analysis may become very difficult.

In Section II of this paper, the fuzzy logic control system considered in this paper will be introduced, and some definitions, useful properties and theorems will be stated. The proposed stability analysis method will be discussed in Section III. An example will be given in Section IV to illustrate the ability and merits of the stability analysis method. The plant in this example has a control signal function composed of piecewise linear functions, saturation functions and a deadband. Finally, a conclusion will be drawn in Section V.

\section{FUZZY LOGIC CONTROL SYSTEMS}

A fuzzy logic control system comprising a plant and an FLC is shown in Fig. 1. The FLC consists of $r$ fuzzy rules. Each fuzzy rule generates an output $u_{i}$ and a degree of membership $\mu_{i} \in[0,1], i=1,2, \ldots \ldots, r$. The overall control signal applying to the plant is a function of $u_{i}$ and $\mu_{i}$. Let the plant be of single input, $n$-th order and non-linear type which can be described by the following equation:

$$
\dot{x}=f(x)+b(x) g(u)+w
$$

where $x=\left[x_{1}, x_{2}, \ldots \ldots, x_{n}\right]^{\mathrm{T}}$ is a state-vector, $f(x)=\left[f_{1}(x), f_{2}(x)\right.$, $\left.\ldots \ldots, f_{n}(\boldsymbol{x})\right]^{\mathrm{T}}$ and $\boldsymbol{b}(\boldsymbol{x})=\left[b_{1}(\boldsymbol{x}), b_{2}(\boldsymbol{x}), \ldots \ldots, b_{n}(\boldsymbol{x})\right]^{\mathrm{T}}$ are function vectors describing the dynamics of the plant, $\boldsymbol{w}=\left[w_{1}, w_{2}, \ldots \ldots\right.$ , $\left.w_{n}\right]^{\mathrm{T}}$ is a vector describing the external disturbances, $g(u)$ is a scalar linear or non-linear function of $u$, and $u$ is a control 


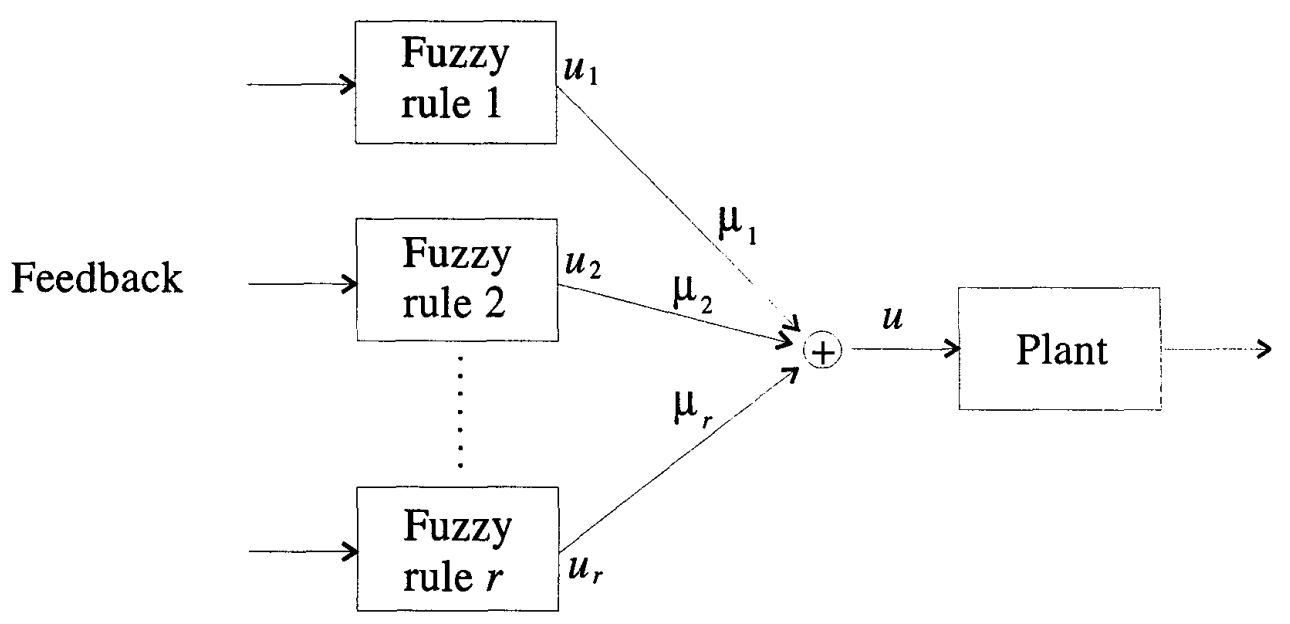

Fig. 1 A general fuzzy logic control system

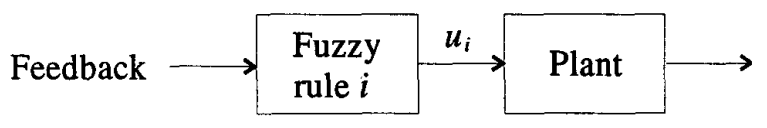

Fig. 2 A fuzzy sub-system

signal generated by an FLC. The $i$-th fuzzy rule of the FLC is of the following form:

$$
\text { Rule } i \text { : IF <premise i> THEN } u=u_{i}
$$

where $<$ premise $i>$ is the premise of fuzzy rule $i$ with an input variable vector $\boldsymbol{z}$. In many cases, $\boldsymbol{z}$ will be the state vector $\boldsymbol{x}$; but it can also be a function of $\boldsymbol{x}$ (e.g. the distance from a sliding plane) $[1,2] . u_{i}$ can be a constant or a function, either linear or non-linear, of $\boldsymbol{x}$. This is an advantage as compared with the case of many existing FLCs that require the rule outputs to be linear state feedback control laws of $x$. It is assumed that for any input $z_{0}$ in the input universe of discourse $Z$, there exists at least one $\mu_{i}$ among all the rules that is non-zero. The membership functions of the output fuzzy sets are assumed to be singletons. The center average defuzzification method is applied and the control signal is given by

$$
u=\frac{\sum_{i=1}^{r} \mu_{i} u_{i}}{\sum_{i=1}^{r} \mu_{i}} .
$$

\subsection{Some definitions and properties}

We shall first define some terms and highlight some useful properties in this sub-section. The Lyapunov theorem on globally asymptotically stability will also be stated.

Definition 1: For any input $z_{o} \in Z$, if the degree of membership $\mu_{i}$ of fuzzy rule $i$ is zero, this fuzzy rule $i$ is called an inactive fuzzy rule for $z_{o}$; otherwise, it is called an active fuzzy rule for $z_{0}$. An active region of a fuzzy rule $i$ is defined as the region $Z_{r} \subset Z$ such that the fuzzy rule $i$ is active for $z \in Z_{r}$.

It should be noted that an inactive fuzzy rule for $z=z_{0}$ will not affect the controller output $u$ (for $z=z_{0}$ ). By letting $I_{A}$ be a set containing the rule numbers of the active fuzzy rules for $z_{o}$, (3) can be rewritten so as to consider all active fuzzy rules (for $z=z_{o}$ ) only:

$$
u=\frac{\sum_{i \in I_{A}} \mu_{i} u_{i}}{\sum_{i \in I_{A}} \mu_{i}} .
$$

Property 1: For any input $z_{o} \in Z$, there exist $p, q \in I_{A}$ such that $u_{p} \leq u_{i} \leq u_{q}$ for all $i \in I_{A}$.

Definition 2: A fuzzy sub-system (as shown in Fig. 2) associated with fuzzy rule $i$ is a system with a plant of (1) controlled by only $u_{i}$, which is the output of fuzzy rule $i$ in the form of (2).

To prove the global asymptotic stability of a non-linear system, the Lyapunov theorem can be applied, which can be stated as follows [3]:

Theorem 1: Assume that there exists a scalar function $V$ of the state $\boldsymbol{x}$, with continuous first order derivatives such that

M11: $V(x)$ is positive definite,

M12: $\dot{V}(x)$ is negative definite,

M13: $V(x) \rightarrow \infty$ as $\|x\| \rightarrow \infty$,

then the equilibrium point at the origin is globally asymptotically stable. 


\section{STABILITY ANALYSIS METHOD}

One major difficulty in analysing the stability of the whole fuzzy logic control system is that we have to tackle a very complex non-linear control $u$. Fig. 1 shows that $u$ is a function of $u_{i}$ and $\mu_{i}$ for $i \in[1, r]$. Moreover, the $\mu_{i}$ 's are functions of $z$. The idea of the proposed stability analysis method is to break down the problem of analysing the stability of the whole fuzzy logic control system into analysing every fuzzy sub-system (as defined in Definition 2) individually. In this case, only one $u_{i}$ instead of many $u_{i}$ 's has to be considered; and the highly complex non-linear function $\mu_{i}$ is not involved. Hence, the complexity of the stability analysis is drastically decreased. Here, the plant is not restricted to be linear with respect to control. The proposed stability analysis method requires $g(u)$ to be a monotonic increasing or decreasing function only. The detail about the stability analysis method can be summarized in the following theorem.

Theorem 2: If

M21: $\boldsymbol{P}$ is a quadratic, symmetric and positive definite matrix, and $V=x^{T} P x \rightarrow \infty$ as $\|x\| \rightarrow \infty$;

M22: $\dot{V}$ is negative definite in every fuzzy sub-system's active region;

M23: $g(u)$ is either a monotonic increasing or a monotonic decreasing function;

M24: the defuzzification method of (3) is applied,

then the equilibrium point at the origin is globally asymptotically stable.

Proof:

From M21,

$$
\begin{gathered}
V=\boldsymbol{x}^{T} \boldsymbol{P} \boldsymbol{x} \\
\dot{V}=\dot{\boldsymbol{x}}^{T} \boldsymbol{P} \boldsymbol{x}+\boldsymbol{x}^{T} \boldsymbol{P} \dot{\boldsymbol{x}} .
\end{gathered}
$$

From (1),

$$
\begin{aligned}
& \left.\dot{V}=(f x)+b(x) g(u)+w)^{T} P x+x^{T} P(f x)+b(x) g(u)+w\right) . \\
& \text { Let } \quad F=(f(x)+w)^{T} P x+x^{T} P(f(x)+w) \text {, } \\
& B=b(x)^{T} P x+x^{T} P b(x), \\
& \text { then } \quad \dot{V}=F+B g(u) \text {. } \\
& \text { From M22, }\left.\quad \dot{V}\right|_{u=u_{q}}=F+B g\left(u_{q}\right)
\end{aligned}
$$

is negative definite, and

$$
\left.\dot{V}\right|_{u=u_{p}}=F+B g\left(u_{p}\right)
$$

is also negative definite.

The system stability can be proved by considering six cases. Case 1 and Case 2 consider a monotonic increasing $g(u)$. Case 3 and Case 4 consider a monotonic decreasing $g(u)$. Case 5 considers the situation when $B$ is zero. All these five cases assume $\boldsymbol{x} \neq \mathbf{0}$. The situation $\boldsymbol{x}=\mathbf{0}$ will be considered in Case 6. M11 and M13 of Theorem $l$ have already been satisfied due to M21. We have to prove that under these 6 cases, if M22 to M24 are satisfied, $\dot{V}$ is also negative definite in the whole state-space so that M12 of Theorem 1 is satisfied.

Case 1: If $g(u)$ is monotonic increasing, $B$ is positive, $\boldsymbol{x} \neq \mathbf{0}$

Let $\quad \dot{V}_{q 1}=F+B g\left(u_{q}\right)$.

It should be noted that by M22 and (10), $\dot{V}_{q 1}$ is negative definite. Now, $g(u)$ is monotonic increasing, $g\left(u_{q}\right) \geq g(u) \forall u \leq u_{q}$. Consequently,

$$
\dot{V}=F+B g(u) \leq \dot{V}_{q 1}<0 \forall u \leq u_{q} \text {. }
$$

Case 2: If $g(u)$ is monotonic increasing, $B$ is negative, $x \neq 0$

$$
\text { Let } \quad \dot{V}_{p 1}=F+B g\left(u_{p}\right)
$$

which is negative definite by M22 and (11). Now, $g(u)$ is monotonic increasing, $g\left(u_{p}\right) \leq g(u) \forall u \geq u_{p}$. Consequently,

$$
\dot{V}=F+B g(u) \leq \dot{V}_{p 1}<0 \forall u \geq u_{p} .
$$

Case 3: If $g(u)$ is monotonic decreasing, $B$ is positive, $x \neq 0$

$$
\text { Let } \quad \dot{V}_{p^{2}}=F+B g\left(u_{p}\right)
$$

which is negative definite by M22 and (11). Now, $g(u)$ is monotonic decreasing, $g\left(u_{p}\right) \geq g(u) \forall u \geq u_{p}$. Consequently,

$$
\dot{V}=F+B g(u) \leq \dot{V}_{p 2}<0 \forall u \geq u_{p} .
$$

Case 4: If $g(u)$ is monotonic decreasing, $B$ is negative, $\boldsymbol{x} \neq \mathbf{0}$

Let $\quad \dot{V}_{q 2}=F+B g\left(u_{q}\right)$

which is negative definite by M22 and (10). Now, $g(u)$ is monotonic decreasing, $g\left(u_{q}\right) \leq g(u) \forall u \leq u_{q}$. Consequently,

$$
\dot{V}=F+B g(u) \leq \dot{V}_{q 2}<0 \forall u \leq u_{q} .
$$

Case 5: If $B$ is zero, $x \neq 0$

From $\mathrm{M} 22, \quad \dot{V}=F<0$

irrespective of the value of $u$.

Case 6: If $x=0$

From (7) and (8), $F=B=0$ if $\boldsymbol{x}=\mathbf{0}$ irrespective of the values of $f(x), b(x)$ and $w$. 
Consequently, $\quad \dot{V}=0$

irrespective of the value of $u$.

Hence, from Case 1 to Case 6, (12) to (17), we have:

if $u_{i} \in\left[u_{p}, u_{q}\right], i \in I_{A}$,

$$
\dot{V}\left\{\begin{array}{l}
<0 \text { if } x \neq 0 \\
=0 \text { if } x=0
\end{array}\right.
$$

which is negative definite.

Moreover, from M24, Property 1, and (4), we have

$$
\begin{gathered}
\frac{\sum_{i \in I_{A}} \mu_{i} u_{p}}{\sum_{i \in I_{A}} \mu_{i}} \leq \frac{\sum_{i \in I_{A}} \mu_{i} u_{i}}{\sum_{i \in I_{A}} \mu_{i}} \leq \frac{\sum_{i \in I_{A}} \mu_{i} u_{q}}{\sum_{i \in I_{A}} \mu_{i}} \\
\Rightarrow u_{p} \frac{\sum_{i \in I_{A}} \mu_{i} \mu_{i}}{\sum_{i \in I_{A}} \mu_{i}} \leq u \leq u_{q} \frac{\sum_{i \in I_{A}} \mu_{i}}{\sum_{i \in I_{A}} \mu_{A}}
\end{gathered}
$$

$\Rightarrow u_{p} \leq u \leq u_{q}$, equality holds when $u_{i}=u_{p}=u_{q}$.

Hence, (19) shows that the overall FLC output indeed lies between $u_{p}$ and $u_{q}$ if $\mathrm{M} 24$ is satisfied, that is the defuzzification method in (3) is employed to derive the overall control signal.

In conclusion, if M21 is satisfied, M11 and M13 of Theorem 1 are satisfied. Also, if M21 to M24 are satisfied which give (18) and (19), M12 of Theorem 1 is satisfied. Hence, Theorem 2 is proved.

\section{ILLUSTRATIVE EXAMPLE}

Consider a plant of the following form:

$$
\frac{d}{d t}\left[\begin{array}{l}
x_{1} \\
x_{2}
\end{array}\right]=\left[\begin{array}{cc}
0 & 1 \\
-2 & -1
\end{array}\right]\left[\begin{array}{l}
x_{1} \\
x_{2}
\end{array}\right]+\left[\begin{array}{l}
0 \\
2
\end{array}\right] g(u)
$$

where $g(u)$ is a piecewise linear function with deadband and saturation as shown in Fig. 3. An FLC with fuzzy rules described by Table 1 and membership functions shown in Fig. 4 is applied to control the plant and the defuzzification method of (3) is used. The proof of system stability is given as follows:

\section{Proof:}

Let $x=\left[\begin{array}{ll}x_{1} & x_{2}\end{array}\right]^{T}$. We select a quadratic and positive definite Lyapunov function as follows:

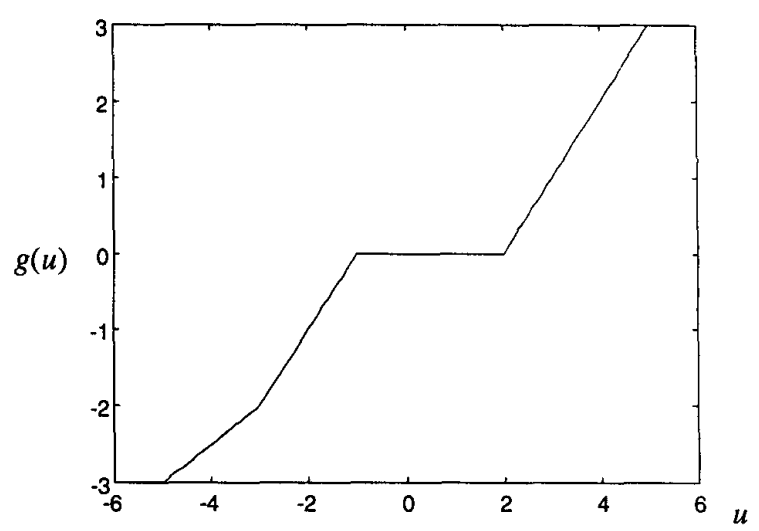

Fig. 3 Function of $g(u)$

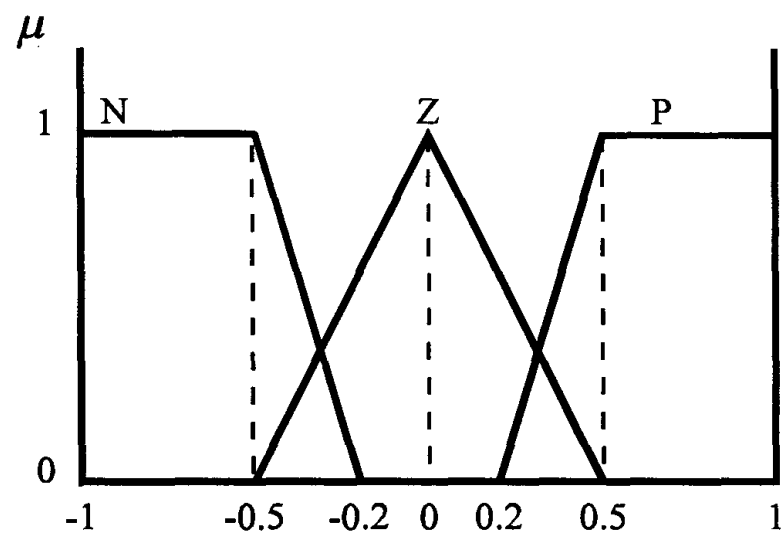

Fig. 4 Membership functions of $x_{1}$ and $x_{2}$

\begin{tabular}{|c|c|c|c|}
\hline Rule & \multicolumn{2}{|c|}{ Antecedent } & Consequent \\
& $x_{1}$ & $x_{2}$ & $u$ \\
\hline 1 & $\mathrm{P}$ & $\mathrm{P}$ & -5 \\
\hline 2 & $\mathrm{~N}$ & $\mathrm{~N}$ & 5 \\
\hline 3 & $\mathrm{Z}$ & $\mathrm{P}$ & -3 \\
\hline 4 & $\mathrm{Z}$ & $\mathrm{N}$ & 3 \\
\hline 5 & $\mathrm{P}$ & $\mathrm{Z}$ & -1 \\
\hline 6 & $\mathrm{~N}$ & $\mathrm{Z}$ & 1 \\
\hline 7 & $\mathrm{P}$ & $\mathrm{N}$ & 0 \\
\hline 8 & $\mathrm{~N}$ & $\mathrm{P}$ & 0 \\
\hline 9 & $\mathrm{Z}$ & $\mathrm{Z}$ & 0 \\
\hline \multicolumn{3}{|c|}{ Table 1 Fuzzy rule base } \\
\\
$\quad V=x^{T}\left[\begin{array}{cc}50 & 1 \\
1 & 25\end{array}\right] x$ \\
$=50 x_{1}^{2}+25 x_{2}^{2}+2 x_{1} x_{2}$.
\end{tabular}

Also $V(x) \rightarrow \infty$ as $\|x\| \rightarrow \infty$. The first derivative of $V$ is given by 


$$
\dot{V}=50 x_{1} \dot{x}_{1}+25 x_{2} \dot{x}_{2}+2 \dot{x}_{1} x_{2}+2 x_{1} \dot{x}_{2} .
$$

From (20),

$$
\begin{aligned}
\dot{V} & =50 x_{1} x_{2}+25 x_{2}\left[-2 x_{1}-x_{2}+2 g(u)\right] \\
& +2 x_{2}^{2}+2 x_{1}\left[-2 x_{1}-x_{2}+2 g(u)\right] \\
& =50 x_{1} x_{2}-50 x_{1} x_{2}-25 x_{2}^{2}+50 x_{2} g(u) \\
& +2 x_{2}^{2}-4 x_{1}^{2}-2 x_{1} x_{2}+4 x_{1} g(u) \\
& =-4 x_{1}^{2}-23 x_{2}^{2}-2 x_{1} x_{2}+4 x_{1} g(u)+50 x_{2} g(u) .
\end{aligned}
$$

For rule $1, x_{1}$ is $\mathrm{P}, x_{2}$ is $\mathrm{P}, u=-5$

$$
\Rightarrow x_{1} \in[0.2,1], x_{2} \in[0.2,1] \text { and } g(u)=-3 \text {. From (22), }
$$

$$
\begin{aligned}
\dot{V} & =-4 x_{1}^{2}-23 x_{2}^{2}-2 x_{1} x_{2}+4 x_{1} g(u)+50 x_{2} g(u) \\
& =-4 x_{1}^{2}-23 x_{2}^{2}-2 x_{1} x_{2}-12 x_{1}-150 x_{2} \\
& <0
\end{aligned}
$$

since $x_{1}$ and $x_{2}$ are positive.

For rule $2, x_{1}$ is $\mathrm{N}, x_{2}$ is $\mathrm{N}, u=5$

$$
\begin{aligned}
& \Rightarrow x_{1} \in[-1,-0.2], x_{2} \in[-1,-0.2] \text { and } g(u)=3 \text {. From }(22), \\
& \qquad \begin{aligned}
\dot{V} & =-4 x_{1}^{2}-23 x_{2}^{2}-2 x_{1} x_{2}+4 x_{1} g(u)+50 x_{2} g(u) \\
& =-4 x_{1}^{2}-23 x_{2}^{2}-2 x_{1} x_{2}+12 x_{1}+150 x_{2} \\
& <0
\end{aligned}
\end{aligned}
$$

since $x_{1}$ and $x_{2}$ are negative.

For rule $3, x_{1}$ is $\mathrm{Z}, x_{2}$ is $\mathrm{P}, u=-3$

$\Rightarrow x_{1} \in[-0.5,0.5], x_{2} \in[0.2,1]$ and $g(u)=-2$. From (22),

$$
\begin{aligned}
\dot{V} & =-4 x_{1}^{2}-23 x_{2}^{2}-2 x_{1} x_{2}-8 x_{1}-100 x_{2} \\
& \leq-4 x_{1}^{2}-23 x_{2}^{2}+1+4-100 x_{2} \\
& <0
\end{aligned}
$$

since $\max \left(5-100 x_{2}\right)=-15$.

For rule $4, x_{1}$ is $\mathrm{Z}, x_{2}$ is $\mathrm{N}, u=3$

$$
\begin{gathered}
\Rightarrow x_{1} \in[-0.5,0.5], x_{2} \in[-1,-0.2] \text { and } g(u)=1 \text {. From }(22), \\
\dot{V}=-4 x_{1}^{2}-23 x_{2}^{2}-2 x_{1} x_{2}+4 x_{1}+50 x_{2} \\
\leq-4 x_{1}^{2}-23 x_{2}^{2}+1+2+50 x_{2} \\
<0
\end{gathered}
$$

since $\max \left(3+50 x_{2}\right)=-7$.

For rule $5, x_{1}$ is $\mathrm{P}, x_{2}$ is $\mathrm{Z}, u=-1$

$$
\begin{aligned}
\Rightarrow x_{1} \in[0.2,1], x_{2} & \in[-0.5,0.5] \text { and } g(u)=0 . \text { From }(22), \\
\dot{V} & =-4 x_{1}^{2}-23 x_{2}^{2}-2 x_{1} x_{2} \\
& =-3 x_{1}^{2}-22 x_{2}^{2}-\left(x_{1}+x_{2}\right)^{2} \\
& <0 .
\end{aligned}
$$

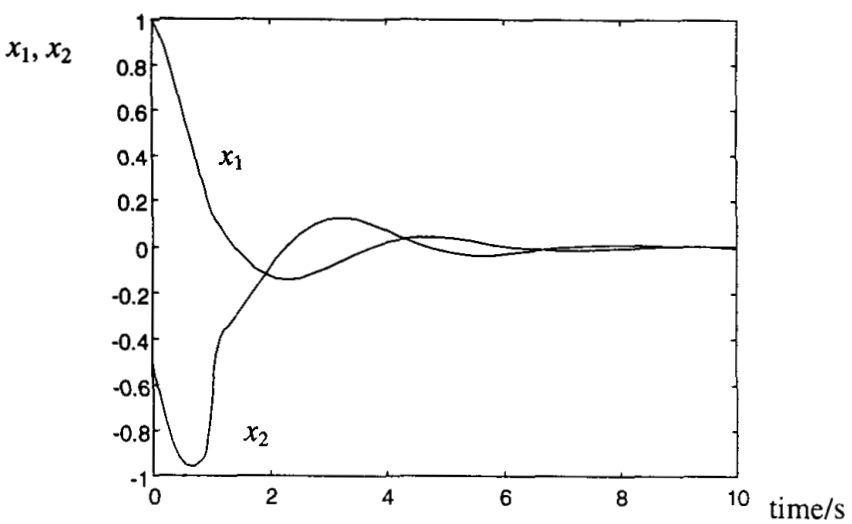

Fig. 5 Responses of the system with non-linear in control

For rule 6 to 8 , since $g(u)=0$, the analysis for rule 5 can also be applied to give the result $\dot{V}<0$.

For rule 9 , if $\left[\begin{array}{ll}x_{1} & x_{2}\end{array}\right] \neq\left[\begin{array}{ll}0 & 0\end{array}\right]$, the analysis of rule 5 can be applied to give the result $\dot{V}<0$. If $\left[\begin{array}{ll}x_{1} & x_{2}\end{array}\right]=\left[\begin{array}{ll}0 & 0\end{array}\right]$, from (22), $\dot{V}=0$.

Hence, $\dot{V}$ is negative definite in every fuzzy sub-system's active region, when $g(u)$ is monotonic increasing and the defuzzification method of (3) is applied. From Theorem 2, the equilibrium point at the origin is globally asymptotically stable.

QED

Simulation results of the zero-input responses of the closed-loop system with initial values $\boldsymbol{x}(0)=\left[\begin{array}{ll}1 & -0.5\end{array}\right]^{\mathrm{T}}$ are shown in Fig. 5. The stability of the fuzzy logic control system is verified.

\section{CONCLUSION}

An improved Lyapunov function based stability analysis method for fuzzy logic control systems has been presented in this paper. This method is advantageous in that every fuzzy sub-system is handled individually so as to reduce drastically the complexity of analysis, the shape and distribution of the membership functions are not restricted to certain patterns so as to make the FLC more flexible, and the plant in the system is not needed to be linear with respect to control. The last point is important because many TS fuzzy model based stability analysis methods are difficult to apply to plants that are not linear with respect to control, as this may lead to a premise depending on the control (in addition to the state) in the fuzzy control rules. This paper has shown how the proposed stability analysis method can be applied to tackle plants that are non-linear with respect to control, and the nonlinear function of control is either monotonic increasing or decreasing. An example has been given to illustrate how the 
method can be applied to a plant with a control signal function which is piecewise linear and with saturation and deadband. It can be seen that the stability analysis is simple.

\section{REFERENCE}

[1] L. K. Wong, F. H. F. Leung, and P. K. S. Tam, "Lyapunov function based design of fuzzy logic controllers and its application on combining controllers," IEEE Trans. Ind. Electronics, vol. 45, no. 3, pp. 502509, June 1998.

[2] L. K. Wong, F. H. F. Leung, and P. K. S. Tam, "Combination of sliding mode controller and PI controller using fuzzy logic controller," in Proc. 1998 IEEE Int. Conf. Fuzzy Syst., Anchorage, Alaska, USA, May 1998, pp. 296-301.

[3] J. J. E. Slotine and W. Li, Applied Nonlinear Control, Prentice-Hall, Inc. Englewood Cliffs, N.J. 1991.

[4] S. G. Cao, N. W. Rees, and G. Feng, "Analysis and design for a class of continuous-time fuzzy control systems," Int. J. Control, vol. 64, pp. 1069-1089, 1996.

[5] S. G. Cao, N. W. Rees, and G. Feng, "Analysis and design for a class of complex control systems, Part II: Fuzzy controller design," Automatica, vol. 33, no. 6, pp. 1029-1039, 1997.

[6] S. C. Cao, N. W. Rees, and G. Feng, "Analysis and design of fuzzy control systems using dynamic fuzzystate space models," IEEE Trans. Fuzzy Systems, vol. 7, no. 2, pp. 192-200, April 1999.

[7] K. Tanaka, T. Ikeda, and H. O. Wang, "Robust stabilization of a class of uncertain nonlinear system via fuzzy control: Quadratic stabilizability, $\mathrm{H}^{\infty}$ control theory, and linear matrix inequalities," IEEE Trans. Fuzzy Systems, vol. 4, no. 1, pp. 1-13, February 1996.

[8] H. O. Wang, K. Tanaka, and M. F. Griffin, "An approach to fuzzy control of nonlinear systems: stability and design issues," IEEE Trans. Fuzzy Systems, vol. 4, no. 1, pp. 14-23, February 1996.

[9] R. K. Mudi and N. R. Pal, "A robust self-tuning scheme for PI- and PD-type fuzzy controllers," IEEE Trans. Fuzzy Systems, vol. 7, no. 1, pp. 2-16, February 1999.

[10] H. Ying, "Practical design of nonlinear fuzzy controllers with stability analysis for regulating processes with unknown mathematical models," Automatica, vol. 30, no. 7, pp. 1185-1195, 1994.

[11] H. Ying, W. Siler, and J. J. Buckley, "Fuzzy control theory: A nonlinear case," Automatica, vol. 26, no. 3, pp. 513-20, 1990.

[12] F. H. F. Leung, H. K. Lam, and P. K. S. Tam, "Design of fuzzy controllers for uncertain nonlinear systems using stability and robustness analyses," System and Control
Letters, vol. 35, no. 4, pp. 237-283, 1998.

[13] M. C. M. Teixeira and S. H. Zak, "Stabilizing controller design for uncertain nonlinear systems using fuzzy models," IEEE Trans. Fuzzy Systems, vol. 7, no. 2, pp. 133-142, April 1999.

[14] V. I. Utkin, "Variable structure systems with sliding modes," IEEE Trans. on Automatic Control, pp. 212222, April 1977.

\section{ACKNOWLEDGEMENT}

The work described in this paper was substantially supported by a grant from the Research Grants Council of the Hong Kong Special Administrative Region (Project No. APA54). 